\title{
Dental pulp stem cells express tendon markers under mechanical loading and are a potential cell source for tissue engineering of tendon-like tissue
}

\author{
Yu-Ying Chen ${ }^{1,2}$, Sheng-Teng He ${ }^{3}$, Fu-Hua Yan ${ }^{4,5}$, Peng-Fei Zhou ${ }^{2}$, Kai Luo ${ }^{2}$, Yan-Ding Zhang ${ }^{6}$, Yin Xiao ${ }^{5,7}$ \\ and Min-Kui Lin ${ }^{2}$ \\ Postnatal mesenchymal stem cells have the capacity to differentiate into multiple cell lineages. This study explored the \\ possibility of dental pulp stem cells (DPSCs) for potential application in tendon tissue engineering. The expression of tendon- \\ related markers such as scleraxis, tenascin-C, tenomodulin, eye absent homologue 2, collagens I and $\mathrm{VI}$ was detected in dental \\ pulp tissue. Interestingly, under mechanical stimulation, these tendon-related markers were significantly enhanced when DPSCs \\ were seeded in aligned polyglycolic acid (PGA) fibre scaffolds. Furthermore, mature tendon-like tissue was formed after \\ transplantation of DPSC-PGA constructs under mechanical loading conditions in a mouse model. This study demonstrates that \\ DPSCs could be a potential stem cell source for tissue engineering of tendon-like tissue.
}

International Journal of Oral Science (2016) 8, 213-222; doi:10.1038/ijos.2016.33; published online 4 November 2016

Keywords: dental pulp stem cells; in vivo model; mechanical loading; tendon engineering

\section{INTRODUCTION}

Tendon development can be divided into the following two stages: cell determination of progenitors and differentiation/maturation. During these stages, some crucial tendon-related markers are involved. ${ }^{1}$ Scleraxis (SCX) is a member of the basic helix-loop-helix superfamily of transcription factors and a relatively specific tendon marker. ${ }^{2}$ Tenascin-C (TNC) binds to tenocyte membrane and provides elasticity to tendons. Eye absent 2 (EYA2), as a muscle transcription factor, has a role in certain aspects of tendon development; thus many researchers have used it to help determine tenogenic differentiation. ${ }^{3}$ TNC and EYA2 are the key target genes of SCX. Tenomodulin (TNMD) is a late differentiation marker of tenocyte and can regulate tenocyte proliferation. ${ }^{4}$ These molecules have critical roles in controlling the fate of tendon cells.

Tendon injuries are difficult to treat and often cause significant dysfunction and disability, leading to instability, abnormal joint movement, and pain. ${ }^{5}$ Traditional treatments can only reduce pain over a long healing phase, and a surgical method may be needed to repair or replace the damaged tendons; however, surgery can induce complications. ${ }^{3-4}$ Stem cells and tissue engineering may be a promising alternative strategy for tendon repair. Recently, a great deal of effort has been exerted to seek suitable stem cells for tendon regeneration.
Postnatal mesenchymal stem cells (MSCs) have been found to be capable of differentiating down tendon-like cell lineages, ${ }^{6-7}$ and their potential for tendon repair has been demonstrated.

The tooth is one of the most accessible organs for tissue banking and collection of stem cells, including dental pulp stem cells (DPSCs), particularly as most of the adult population might need wisdom tooth extraction. ${ }^{8}$ DPSCs have been widely investigated for their potential in treating various degenerative diseases, such as Alzheimer's disease, myocardial infarction, bone defects, muscular dystrophy, and corneal reconstruction..$^{9-10}$ However, whether DPSCs can serve as a potential cell source for tendon tissue repair and regeneration has not yet been explored.

Dental pulp in the tooth pulp chamber is a loose connective tissue originating from neural crest cells. The pulp includes the odontogenic zone and the pulp proper, and the latter consists primarily of fibroblasts, extracellular matrix (ECM), blood vessels, and nerves. Dental pulp contains collagen fibres synthesized and secreted mainly by fibroblasts or undifferentiated pulp cells (the major cell type), especially in the case of collagen I and collagen VI. ${ }^{11}$ Dental pulp also contains some non-collagenous proteins (NCPs), such as decorin and biglycan. Collagen I, collagen VI, and certain NCPs are also important tendon-related proteins; therefore, this study investigated tendon-

${ }^{1}$ Department of Stomatology, Fujian Provincial Hospital, Fuzhou, China; ${ }^{2}$ School and Hospital of Stomatology, Fujian Medical University, Fuzhou, China; ${ }^{3}$ Department of Stomatology, Hainan Province Nongken Sanya Hospital, Sanya, China; ${ }^{4}$ Nanjing Stomatological Hospital, Medical School of Nanjing University, Nanjing, China; ${ }^{5}$ Australia-China Centre for Tissue Engineering and Regenerative Medicine, Queensland University of Technology, Brisbane, Australia; ${ }^{6}$ College of Life Science, Fujian Normal University, Fuzhou, China and ${ }^{7}$ Institute of Health and Biomedical Innovation, Queensland University of Technology, Brisbane, Australia

Correspondence: Professor MK Lin, School and Hospital of Stomatology, Fujian Medical University, 246 Yangqiao Zhong Road, Fuzhou 350002, China

E-mail: linmk105@sina.com

Professor FH Yan, Nanjing Stomatological Hospital, Medical School of Nanjing University, 30 Zhongyang Road, Nanjing 210008, China

E-mail: fhyan2005@126.com

Accepted 7 June 2016 
related protein expression in dental pulp and tendon-like tissue reconstruction using DPSCs.

\section{MATERIALS AND METHODS}

\section{Cell isolation and culture}

The protocols used were approved by the Human Ethics Committee of Fujian Medical University. Human DPSCs were isolated and cultured according to previously published work. ${ }^{8}$ In this study, normal human impacted third molars or premolars were obtained from 10 patients (11-25 years of age) at the Department of Oral and Maxillofacial Surgery, School and Hospital of Stomatology, Fujian Medical University. Teeth surfaces were cleaned with iodine and phosphate-buffered saline (PBS), and the teeth were broken into pieces to expose the pulp chambers. Pulp tissue was gently separated from the crown and root. The entire tissue was cut into tiny pieces using a surgical knife and then digested in a solution of $3 \mathrm{mg} \cdot \mathrm{mL}^{-1}$ collagenase type I and $4 \mathrm{mg} \cdot \mathrm{mL}^{-1}$ dispase III (Sigma-Aldrich, St Louis, MO, USA) for $30 \mathrm{~min}$ at $37^{\circ} \mathrm{C}$. Single-cell suspensions were obtained by passing the cells through a $70 \mu \mathrm{m}$ strainer (BD Biosciences, San Jose, CA, USA) and then seeding into $6 \mathrm{~cm}$ plates (Nunc, Thermo Fisher Scientific, Waltham, MA, USA) grown with $\alpha$-modified Eagle medium ( $\alpha$-MEM; Hyclone, Beijing, China) supplemented with $10 \%$ foetal bovine serum (FBS; Hyclone, Logan, UT, USA), $100 \mathrm{U} \cdot \mathrm{mL}^{-1}$ penicillin, and $100 \mu \mathrm{g} \cdot \mathrm{mL}^{-1}$ streptomycin (Hyclone, Logan, UT, USA). The culture medium was refreshed the next day.

\section{Polyclonal selection}

DPSCs at the first passage were seeded at a low density of 2 cells per $\mathrm{cm}^{2}$ to form colonies. The medium was replaced every 3 days. After 12 days, the colonies were visualized with $1 \%$ crystal violet/methanol staining for $10 \mathrm{~min}$. Single colonies with diameter $>2 \mathrm{~mm}$ were counted. In the case of small cell clusters, a cluster of $>25$ cells was considered a colony. All DPSCs in this study are of polyclonal origin. The cells were trypsinized when they reached 90\% confluency and were split at a passage ratio of 1:4. Cells between passages 1 and 4 were used in the experiments.

\section{In vitro multipotency assays}

To evaluate their ability to differentiate into other cell types, DPSCs were seeded into six-well plates at a density of $3 \times 10^{5}$ cells per well. The cells were cultured in different media upon reaching $90 \%$ confluence. For the osteogenic differentiation assay, the cell culture medium was supplemented with $15 \%$ FBS, $10 \mathrm{mmol} \cdot \mathrm{L}^{-1} 10 \%$ $\beta$-glycerophosphate, $5 \mathrm{mg} \cdot \mathrm{mL}^{-1}$ ascorbic acid 2-phosphate, and $10^{-5} \mathrm{~mol} \cdot \mathrm{L}^{-1} \quad 0.1 \%$ dexamethasone for 4 weeks of induction. For adipogenic differentiation, a mixture including $0.5 \mathrm{mmol} \cdot \mathrm{L}^{-1}$ isobutylmethylxanthine, $0.5 \mathrm{mmol} \cdot \mathrm{L}^{-1}$ hydrocortisone, $60 \mu \mathrm{mol} \cdot \mathrm{L}^{-1}$ indomethacin, and 15\% FBS was added before culturing for 4 weeks. To induce chondrogenic differentiation, cell pellets (of $2.5 \times 10^{5}$ cells per pellet) were cultured in chondrogenic differentiation media (Gibco, Carlsbad, CA, USA) for 4 weeks. After the induction periods, cells or cell pellets were fixed in $4 \%$ paraformaldehyde for $20 \mathrm{~min}$. The degrees of osteogenic, adipogenic, and chondrogenic differentiation were determined by Alizarin red staining, the formation of lipid vacuoles (visualized by Oil red $\mathrm{O}$ staining according to standard protocols), and Alcian blue staining, respectively. Each test was repeated at least three times.

\section{Fluorescence-activated cell sorting analysis}

Cells obtained from passage 1 were characterized using flow cytometry to determine their cell surface markers. In this part of the experiment, normal human impacted third molars or premolars were obtained from five individual patients. Trypsin/ethylenediaminetetraacetic acid $(0.25 \%)$ was used to detach cells from the culture dish and to dissociate cell aggregates. The cells were placed into a conical centrifuge tube, and cell count and viability analyses were performed. Cells were centrifuged and resuspended with an appropriate volume to achieve a final cell concentration of $1 \times 10^{6} \mathrm{~mL}^{-1}$. To block nonspecific Fc-mediated interactions, the cells were preincubated with $20 \mu \mathrm{L}$ of affinity purified human FcyR-binding inhibitor for $30 \mathrm{~min}$ on ice prior to staining. The anti-Strol-phycoerythrin (PE) mouse monoclonal antibody (Novus Biologicals, Littleton, CO, USA) and anti-CD146 PE mouse monoclonal antibody (eBioscience, San Diego, CA, USA) were incubated for $1 \mathrm{~h}$ in the dark on ice. Mouse anti-human antibodies of CD29-FITC, CD34-FITC, and CD44-FITC (BD Biosciences, San Jose, CA, USA) were also used for fluorescenceactivated cell sorting (FACS) analysis. The cells were washed twice with flow cytometry staining buffer. Mouse isotype antibodies served as controls. The samples were analysed using the BD CellQuest Pro software (BD Biosciences, San Jose, CA, USA). The results were analysed using the FCS Express V3 software (Thornhill, ON, Canada).

\section{Cell proliferation assay}

DPSCs at passage 3 were seeded into 96 -well plates at a density of $5 \times 10^{3}$ cells per well. After $24 \mathrm{~h}$, culture medium with $20 \mu \mathrm{L}$ of 3-[4,5-dimethylthiazol-2-yl]-2,5 diphenyl tetrazolium bromide (MTT) reagent was added (final concentration $0.5 \mathrm{mg} \cdot \mathrm{mL}^{-1}$; Beyotime Institute of Biotechnology, Shanghai, China) according to the instructions of the manufacturer and then incubated at $37^{\circ} \mathrm{C}$. After $4 \mathrm{~h}$, the cells were washed and the formazan derivative was dissolved in $150 \mathrm{~mL}$ dimethyl sulphoxide (Sigma-Aldrich, St Louis, MO, USA). The MTT reduction/attenuation values for each well were measured spectrophotometrically at $490 \mathrm{~nm}$. The MTT assay was carried out every day for 8 days and repeated in triplicate.

For the cell cycle analysis, DPSCs at passage 3 were detached and resuspended at $1 \times 10^{6}$ cells per tube with $70 \%$ cold alcohol and then at $4{ }^{\circ} \mathrm{C}$ for $24 \mathrm{~h}$ for fixation. The next day, the cell suspension was centrifuged at $1000 \mathrm{r} \cdot \mathrm{min}^{-1}$ for $5 \mathrm{~min}$, washed with PBS, centrifuged, resuspended in $0.1 \mathrm{~mL}$ PBS containing $1 \mu \mathrm{L}$ of $10 \mathrm{mg} \cdot \mathrm{mL}^{-1} \mathrm{RNase}$, and incubated at $37^{\circ} \mathrm{C}$ for $30 \mathrm{~min}$. The cells were then stained with propidium iodide by adding $0.4 \mathrm{~mL}$ of $0.05 \mathrm{mg} \cdot \mathrm{mL}$ (Sigma-Aldrich, St Louis, MO, USA) for $30 \mathrm{~min}$ on ice. The cells were analysed by flow cytometry (BD Biosciences, San Jose, CA, USA). Histogram plots were created using the CellQuest software (Beckton Dickinson, San Jose, CA, Canada). The percentages of cells within the various phases of the cell cycle were calculated using the FCS Express V3 software. The fraction of cells in the $G_{1}, S, G_{2}$, and $M$ phases of the cell cycle was quantified. Three separate experiments were performed.

\section{Characterization of dental pulp tissue and tendon}

Normal human discarded tendons were obtained from healthy adults undergoing autologous hamstring tendon transplantation after obtaining informed consent from each patient. Fresh human dental pulp tissues and tendons were trimmed and immediately fixed in $4 \%$ paraformaldehyde solution, dehydrated using an alcohol gradient, and embedded within paraffin blocks for the 
following experiments. Histological sections $(5 \mu \mathrm{m})$ were prepared using a microtome and subsequently stained with haematoxylin and eosin (H\&E) stain.

For immunohistochemical analysis, deparaffinized sections were washed and a heat-mediated antigen retrieval step in citrate buffer was performed, and then non-specific endogenous peroxidase activity was quenched by immersion in $3 \% \mathrm{H}_{2} \mathrm{O}_{2}$ for $15 \mathrm{~min}$. The expression of tendon-related protein was detected by the following antibodies: anti-collagen I (1:500 in PBS; Abcam, Cambridge, MA, USA), anticollagen VI (1:100 in PBS; Abcam, Cambridge, MA, USA), anti-SCX (1:50 in PBS; Abcam, Cambridge, MA, USA), anti-EYA2 (1:500 in PBS; Abcam, Cambridge, MA, USA), anti-TNMD (1:50 in PBS; Santa Cruz Biotechnology, Santa Cruz, CA, USA), and anti-TNC (1:50 in PBS; Abcam, Cambridge, MA, USA). Sections were first incubated with primary antibody at $4{ }^{\circ} \mathrm{C}$ overnight and then with goat anti-rabbit secondary antibody (ZSGB-BIO, Beijing, China) for $30 \mathrm{~min}$ at room temperature, followed by horseradish peroxidase complex (ZSGB$\mathrm{BIO}$, Beijing, China) for $30 \mathrm{~min}$. Positive staining was visualized by diaminobenzidine (ZSGB-BIO, Beijing, China). The sections were counterstained in haematoxylin. A negative control was obtained by performing the same procedure without primary antibodies under identical conditions.

\section{Preparation of cell-scaffold constructs under static mechanical stimulation}

Long polyglycolic acid (PGA) fibres with a diameter of approximately $15-20 \mu \mathrm{m}$ were provided by Shanghai Jurui Biomaterials Company (Shanghai, China). PGA scaffolds were fabricated according to the protocols from the National Tissue Engineering Research and Development Center, China. PGA fibres (100 mg, 15-20 $\mu \mathrm{m}$ diameter) were woven into a cord shape with a length of $5 \mathrm{~cm}$ and a width of $0.3 \mathrm{~cm}$ and then secured on a custom-fabricated spring formed with a stainless steel frame under static tension. In the loaded groups, PGA constructs were fixed on the spring with maximum tensile force (with the strength being provided by a dental arch expansion appliance) applied empirically to a point just below the failure load. The nonloaded group constructs were fixed on the spring with no tension. PGA constructs were sterilized by soaking in $75 \%$ alcohol for $2 \mathrm{~h}$; the alcohol was changed once in the intermediate phase. Afterward, the scaffold was washed with PBS three times and preincubated in $\alpha$-MEM supplemented with $10 \%$ FBS at $37^{\circ} \mathrm{C}$. After 3 days, the medium was removed and the construct was subsequently air dried for 30 min under ultraviolet light. DPSCs $\left(1 \times 10^{7}\right.$ cells $)$ were seeded onto the scaffold and allowed to completely adhere to them in culture medium for up to $4 \mathrm{~h}$. The construct was then immersed in an adequate culture medium, followed by static culture, with the medium being changed every other day.

\section{Scanning electron microscope examination}

After 1 week of in vitro culture, three of the cell-scaffold constructs were examined by scanning electron microscope (SEM) to visualize the cell morphology and distribution. ${ }^{6}$

Gene expression analysis by real-time polymerase chain reaction Cell-scaffold constructs (under static tension or no tension) were cultured for 1-3 days ( $n=3$ for both the loaded and non-loaded groups at each time point), and then total RNA was extracted from each sample using the TRIzol reagent (Invitrogen, Carlsbad, CA, USA). Polymerase chain reaction (PCR) was performed as described ${ }^{6}$ using a real-time (RT) PCR cycler (ABI StepOnePlus Real-Time PCR System; Thermo Fisher Scientific, Waltham, MA, USA). The expression levels of
Table 1 The sequences of specific primers for real-time PCR operation

\begin{tabular}{llcc}
\hline Gene & \multicolumn{1}{c}{ Prime sequence $\left(5^{\prime} \rightarrow 3^{\prime}\right)$} & $\begin{array}{c}\text { Product } \\
\text { size/bp }\end{array}$ & $\begin{array}{c}\text { Annealing } \\
\text { temperature } /{ }^{\circ} \mathrm{C}\end{array}$ \\
\hline Col I & $\begin{array}{l}\text { Forward: GGTGATGCTGGTCCTGTTG } \\
\text { Reverse: CATCGTGAGCCTTCTCTTGAG }\end{array}$ & 121 & 60 \\
Col VI & $\begin{array}{l}\text { Forward: GCTGGAGGATGCTGTGAAC } \\
\text { Reverse: CGTGGCGATGATGCTCAG }\end{array}$ & 103 & 60 \\
SCX & $\begin{array}{l}\text { Forward: AGAACACCCAGCCCAAACA } \\
\text { Reverse: TCCTTGCTCAACTTTCTCTGGT }\end{array}$ & 64 & 60 \\
TNC & $\begin{array}{l}\text { Forward: ACCGTCTCTTCCGTCACTTCT } \\
\text { TNMD }\end{array}$ & 122 & 60 \\
Reverse: AACAACTTAGGACAATGCGTCT & $\begin{array}{l}\text { Forward: TCACGCCAGACAAGCAAGT } \\
\text { Reverse: CGTCCTCCTTGGTAGCAGTATG }\end{array}$ & 192 & 60 \\
EYA2 & $\begin{array}{l}\text { Forward: GTGTGGAGAGGAGCAAGGA } \\
\text { Reverse: GTGAGATGGCAGGTGGAGAT }\end{array}$ & 143 & 60 \\
B-Actin & $\begin{array}{l}\text { Forward: GGACCTGACTGACTACCTCAT } \\
\text { Reverse: CGTAGCACAGCTTCTCCTTAAT }\end{array}$ & 107 & 60 \\
\hline EYA2, eye absent 2; PCR, polymerase chain reaction; SCX, scleraxis; TNC,
\end{tabular}
tenascin-C; TNMD, tenomodulin.

the tendon/ligament-related genes in these two groups were monitored based on several related markers, including tendon/ligament-related markers (collagens I and VI, SCX, TNC, EYA2, and TNMD). The designed primers for real-time PCR analyses are listed in Table 1. $\beta$-Actin was used as a standard housekeeping gene to normalize the mRNA levels. Real-time PCR was conducted in triplicate for each sample, and the experiments were repeatedly performed using three cell scaffolds.

\section{In vivo implantation of cell-scaffold constructs}

Nude mice (8-12 weeks old) were purchased from Shanghai Laboratory Animal Center National Rodent Laboratory Animal Resources (Shanghai, China). Twenty nude mice were used for in vivo implantation ( $n=5$ for both the loaded and non-loaded groups at each time point of 8 and 14 weeks). All of the experimental protocols were approved by the Animal Care and Experiment Committee of Fujian Medical University. To determine the potential for tendon formation, the DPSC-scaffold constructs were implanted subcutaneously in the nude mice under mechanical loading conditions, with each mouse receiving one cell construct. Briefly, 20 male mice were randomly divided into two groups. Each mouse was anaesthetized by intraperitoneal injection of $0.1 \mathrm{~mL} 10 \%$ chloral hydrate followed by dorsal skin incision, and then a subcutaneous pocket was created to expose the dorsal muscularis fascia. In the loaded group, the cell-scaffold constructs (DPSC-PGA) $(5 \mathrm{~cm})$ were sutured to the fascia nuchae cranially and to the fascia of the musculi sacrospinalis with Ethilon 6-0 sutures (Johnson \& Johnson, New Brunswick, NJ, USA) to provide in vivo mechanical loading. For the cell-scaffold constructs without loading, the constructs were simply implanted into the subcutaneous pocket without suturing. Therefore, the natural movement of the mouse provided cyclic mechanical loading on the appropriate cell-scaffold constructs. At the time points of 8 or 14 weeks, the animals were killed, and the implanted tissues were harvested for the following analyses. 
Gross examination, histology, and immunohistochemical staining Samples harvested from the two groups were compared with each other in terms of their tissue colour, appearance, vascularization, and histological and immunohistochemical examinations. All samples were fixed in $4 \%$ paraformaldehyde for $24 \mathrm{~h}$, dehydrated in an ascending series of alcohol concentration, embedded in paraffin, and then sectioned to $5 \mu \mathrm{m}$ thickness. Sections were stained with H\&E and Masson trichrome. A polarizing microscope was used to examine the H\&E-stained tissue samples to determine the collagen structure and PGA degradation in the formed tissues of different groups. To verify the human origin of engineered tendon-like tissues, immunostaining of HLA class I by the primary monoclonal anti-HLA class I antibody EMR8-5 (1:200 in PBS; Abcam, Cambridge, MA, USA) was conducted. The protocol was the same as described above, and tendon-related protein expression was also characterized.

\section{Statistical analysis}

Data were analysed using the GraphPad Prism 5 software (La Jolla, CA, USA). Within-group differences were analysed with Student's $t$-test at a particular time point. Additionally, one-way analysis of variance test was performed to analyse the differences in the expression of tendon-related genes, as well as the differences in tendon width and thickness, among different time points. A level of $P<0.05$ was considered statistically significant.

\section{RESULTS}

Identification and characterization of DPSCs

Primary human DPSCs extracted from enzyme digestion were round shaped, adhered to culture dishes strongly, formed adherent colonies, and displayed fibroblast-like morphology after 7 days in culture (Supplementary Data S1A) and formed larger colonies after 12 days in culture. Approximately $40 \%$ of DPSCs at passage 1 were able to form colonies. Cell differentiation potential assays further revealed that DPSC pellets exhibited strong blue staining for cartilage ECM after chondrogenic induction (Supplementary Data S1B). DPSCs also formed extensive amounts of Alizarin red-positive mineral deposits throughout the adherent layers (Supplementary Data S1C) during osteogenic differentiation media; they also differentiated into adipocytes, as visualized by Oil red $\mathrm{O}$ staining of intracellular lipid clusters (Supplementary Data S1D). FACS on in vitroexpanded DPSCs indicated the expression of MSC markers, such as CD146 (91.94\%) and Stro-1 (13.13\%) (Supplementary Data S1E and S1F) (other MSC markers were shown in Supplementary Data S2). MTT assays revealed a significantly increased optical density in DPSCs from day 3 to 6 (Supplementary Data S1G). Moreover, the percentage of DPSCs in the $S+G_{2} / M$ phase was 35.61\% (Supplementary Data S1H).

\section{Expression of tendon-related markers in dental pulp tissue and DPSCs}

Immunohistochemical staining was performed on human native dental pulp tissue with antibodies against collagens I and VI, SCX, TNC, EYA2, and TNMD. Surprisingly, SCX, TNC, EYA2, and TNMD were expressed in odontoblastic layers but only moderately positively in the cell-rich zone and weakly in the pulpal core. Collagens I and VI were expressed in the entire dental pulp tissue. In the DPSC clonogenic populations, as shown in Figure 1b, immunofluorescent staining revealed that DPSCs express collagens I and VI but rarely express SCX, TNC, EYA2, or TNMD.
Phase-contrast microscopy and SEM examination of cell-scaffold construct

Passage 3 cultured DPSCs exhibited a spindle and fibroblast-like morphology in 2-D culture dishes and always grew in a monolayer with fewer matrices than in a 3-D scaffold (Figure 2a and 2c). After in vitro culture of the cell-PGA scaffold construct for 7 days, microscopic observation indicated that DPSCs spread along PGA fibres and produced abundant matrices, forming a net-like structure on the PGA scaffold (Figure 2c). SEM examination further demonstrated that DPSCs bound tightly to and spread well on the PGA fibres and produced extracellular matrices around the polymer fibres (Figure 2d), indicating good cytocompatibility of PGA scaffolds for DPSCs.

\section{Mechanical stretch promotes tendon differentiation of DPSCs in vitro}

We investigated the effect of PGA scaffold under mechanical stretch on tenogenic differentiation on DPSCs in vitro. The expression levels of tendon-related ECM genes, transcription factors, and surface markers were analysed using tendon differentiation markers (collagen I and collagen VI, SCX, TNC, EYA2, and TNMD). As expected, collagens I and VI, which are tendon-related ECM genes, were expressed significantly more in the mechanically loaded groups compared with the controls. The same trend was also observed in SCX and SCX-dependent transcription factors (TNC and EYA2) (Figure 2e) ( $P$-values are provided in Supplementary Data S3). However, the tendon marker TNMD, which is also a downstream molecule of SCX, could not be detected in either the loaded or unloaded groups (data not shown).

\section{Macrographic examination of engineered tendons}

To study the effect of mechanical stimuli on tendon-like tissue formation by DPSC-PGA scaffold in vivo, 20 DPSC-PGA constructs engineered in vitro were cultured for 2 weeks under static strain and then implanted in vivo. At 8 weeks postimplantation, both mechanically loaded and unloaded groups presented yellowish colour and cord-like structure with grossly visible PGA residues at the back of the mouse. The non-loaded tendons were thinner, and their width varied from the top to the end. After 14 weeks, the loaded tissue constructs appeared much thicker compared with those not exposed to stretch loading. In addition, the loaded tissue constructs exhibited a smooth and thick surface with more neovascularization along the length of the formed tissue (that is, tendon-like structure). As a whole, the DPSCPGA scaffolds exposed to mechanical loading exhibited a significantly thicker tendon-like structure after 14 weeks (Figure 3a1 and 3a2) compared with the non-loaded tissue (Figure $3 \mathrm{a} 3$ and 3a4). As shown in Figure $3 \mathrm{~b}$ and $3 \mathrm{c}$, the central region of the in vivo-loaded tendon at 14 weeks was the widest $(2.496 \pm 0.172 \mathrm{~mm})$ and the thickest $(3.058 \pm 0.109 \mathrm{~mm})$ among all groups. Statistical analysis indicated a significant difference in width and thickness among all groups $(P<0.01 ; P$-values are provided in Supplementary Data S4) within the different period, further indicating that in vivo mechanical loading has a significant effect on engineered tendon development and maturation.

\section{Histology and polarized microscopic views of engineered DPSC-PGA scaffolds}

$\mathrm{H} \& \mathrm{E}$ and Masson staining revealed an immature tissue structure and high cellular content in the tendon-like tissues that were in vivo engineered for 8 weeks in both groups. In the group with mechanical loading (Figure 4a1), the effect of mechanical stimulus 
a

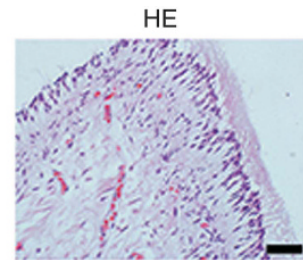

Col VI

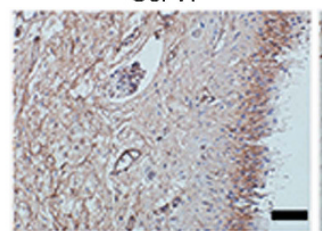

Col I
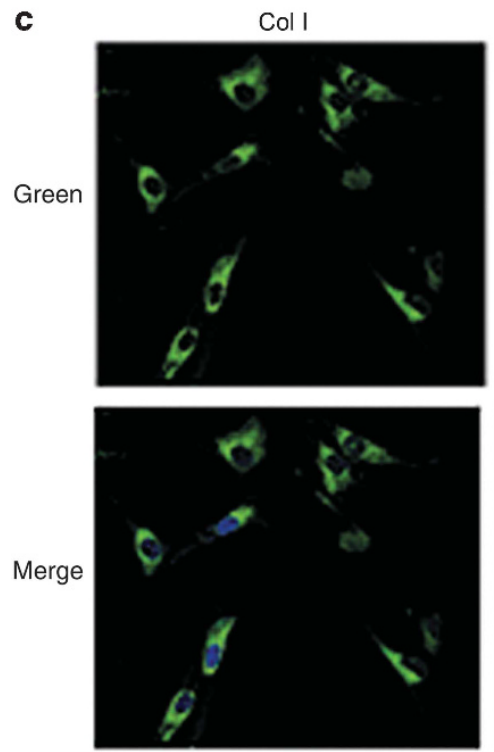

TNC
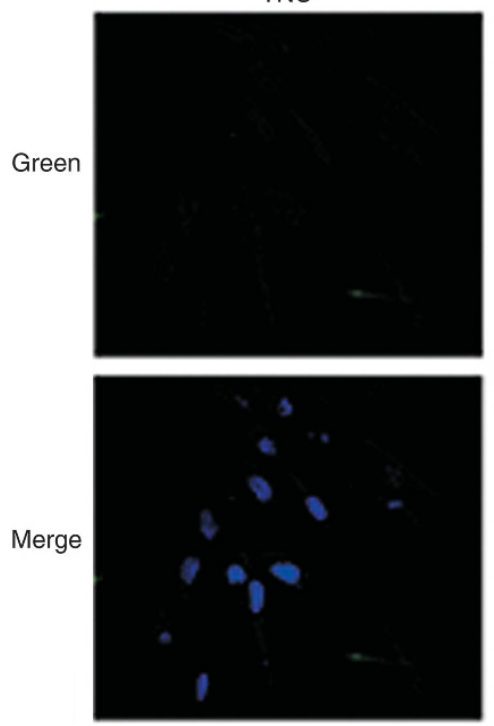

Col I

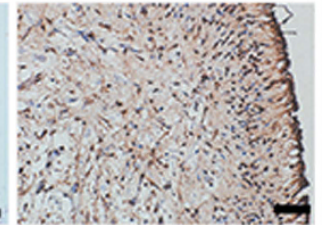

SCX

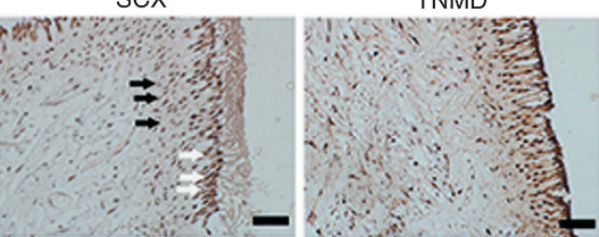

TNC

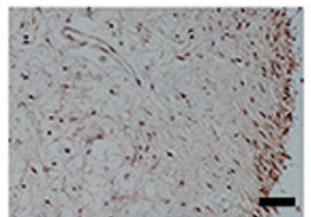

TNMD
Col VI
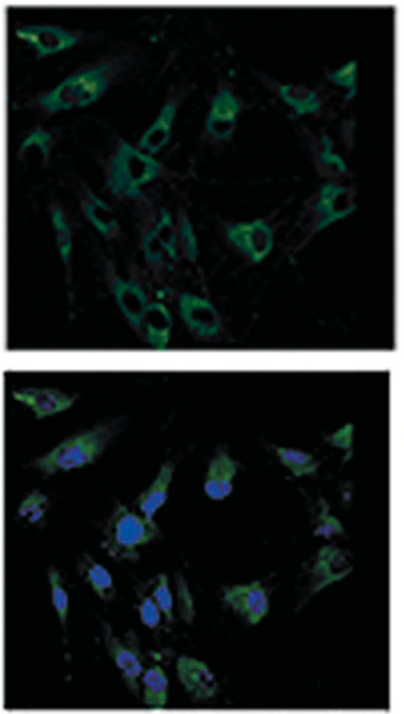

EYA2
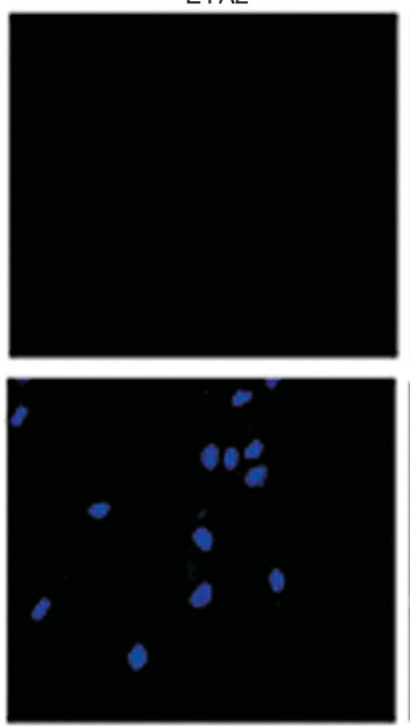

EYA2

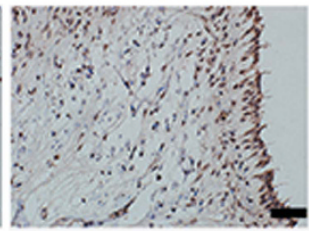

b

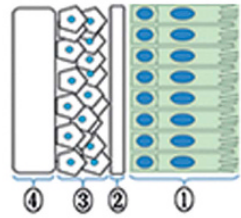

SCX
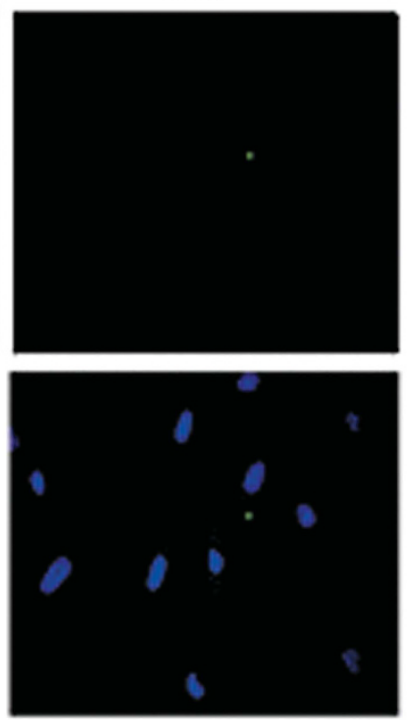

TNMD
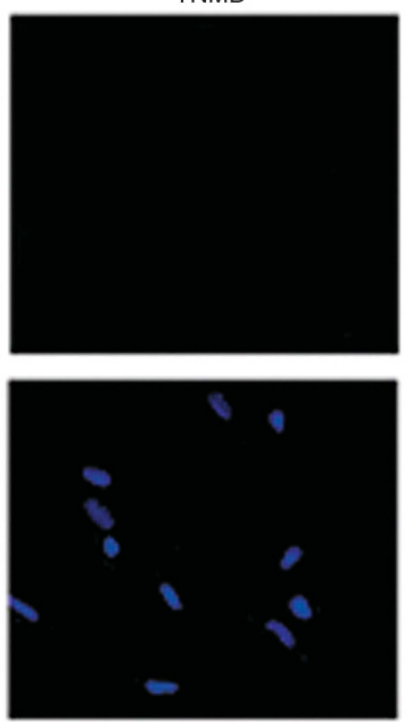

Figure 1 Protein expression of tendon-related molecules in human native dental pulp tissue. (a) Immunohistochemical staining. SCX, TNC, EYA2, and TNMD were expressed in odontoblastic layers (white arrows in the representative picture for SCX) but weakly in the cell-rich zone (black arrows in the representative picture for SCX). Collagens I and VI were apparently expressed in the entire dental pulp tissue. Original magnification: $\times 400$; bar $=50 \mu \mathrm{m}$. (b) Schematic of dental pulp anatomy. (1): odontoblast layer; (2): cell free zone; (3): cell rich zone; (4): pulpal core. (c) Immunofluorescent staining in DPSCs with 4',6-diamidino-2-phenylindole (DAPI) nuclear counterstaining. DPSCs express collagens I and VI, but rarely express SCX, TNC, EYA2, and TNMD. Original magnification: $\times 200$; bar $=25 \mu \mathrm{m}$. DPSCs, dental pulp stem cells; EYA2, eye absent 2 ; SCX, scleraxis; TNC, tenascin-C; TNMD, tenomodulin. 

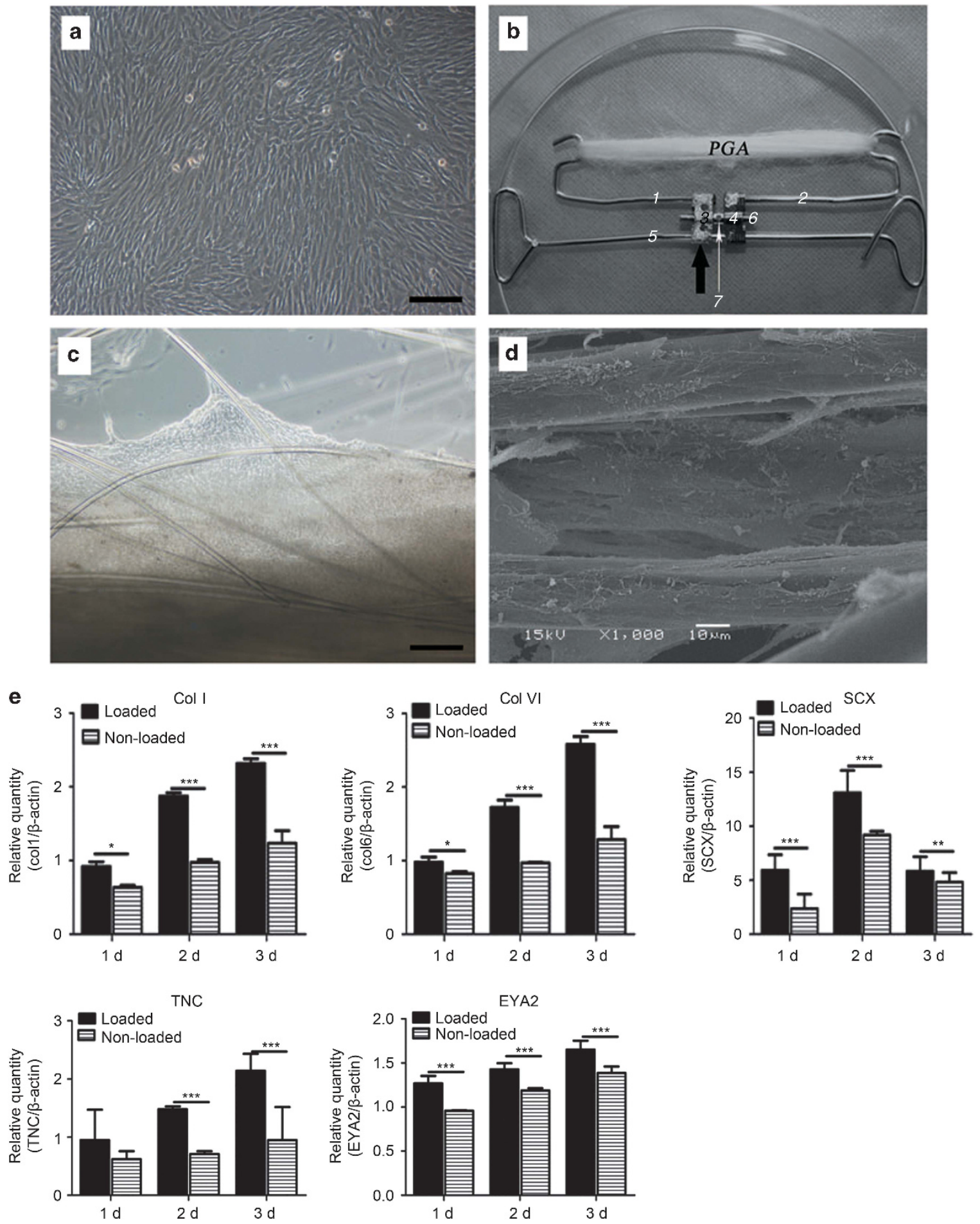

Figure 2 In vitro culture of cell scaffold constructs. Design of tendon complex scaffold. (a) Passage 3 DPSCs cultured for confluence. (b) PGA long fibres were hooked on a custom-fabricated spring. Dark arrow points to a commercial dental arch expansion appliance (including flanks 3 and 4 and central screw axis 6). Wires 1 and 2 are fused onto flanks 3 and 4, respectively. Flanks 3 and 4 can be finely expanded along screw axis 6 by turning a hole in it, and these three parts as a whole can slide along wire 5; the latter constrains the entire appliance in the cell culture dish using two loops. (c) Phase-contrast microscopy of a cell-scaffold construct cultured for 1 week. (d) SEM view shows attachment and spreading of DPSCs on PGA fibres and their matrix production. Original magnifications: (a, c) $\times 100$; bar $=200 \mu \mathrm{m},(\mathrm{d}) \times 1000$, bar $=10 \mu \mathrm{m}$. (e) Quantitative real-time PCR confirms the gene expression of tendon-specific and tendon-related matrix genes in engineered tendon scaffolds with or without loading after 1-3 days $(n=3$, mean \pm SD). Gene expression levels are normalized to the reference gene $\beta$-actin ( $y$ axis); the mRNA harvested from DPSCs was cultured in a dish as a control group. ${ }^{*} P<0.05$ between two groups; $* * P<0.01$ between two groups; ${ }^{* *} P<0.001$ between two groups $(P$-values are provided in Supplementary Data S3). DPSC, dental pulp stem cell; PCR, polymerase chain reaction; PGA, polyglycolic acid; SD, standard deviation; SEM, scanning electron microscope. 
a
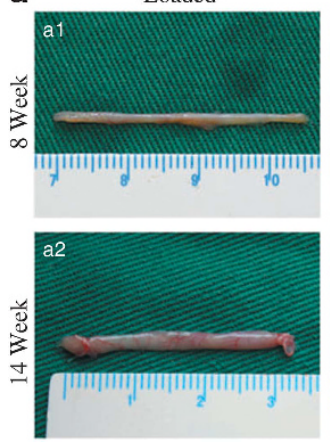

Non-loaded
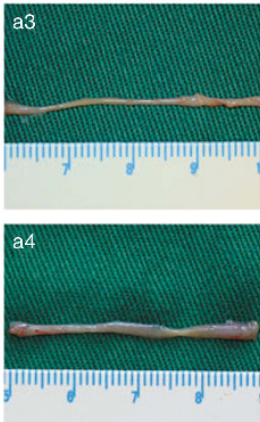

b

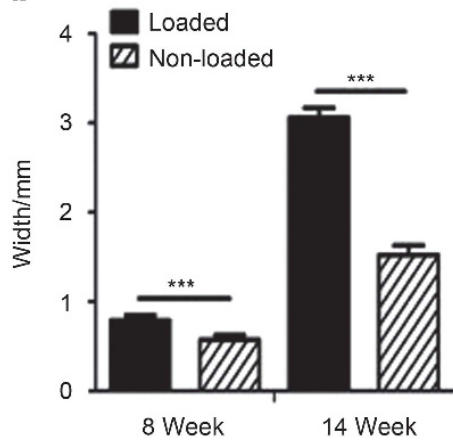

c

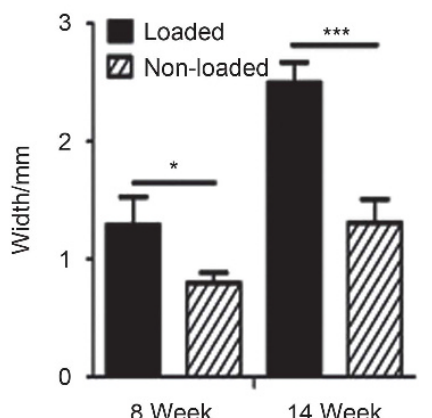

Figure 3 Gross view and dimension analyses of in vivo engineered tendons. (a) Gross view of DPSC-engineered tendon complexes at weeks 8 and 14 after implantation. After 2 weeks of static stretch in vitro, the engineered tendon was transplanted into the mice with (a1, a2) and without (a3, a4) mechanical loading for 8 and 14 weeks. (b, c) Quantitative analyses of engineered tendon complexes on central thickness and width. There are significant differences in these parameters among different groups $(P<0.01)(n=5 ; P$-values are provided in Supplementary Data S4). * and *** respectively indicates significant difference between loaded and non-loaded groups with $P<0.05$ and $P<0.001$. DPSCs, dental pulp stem cells.
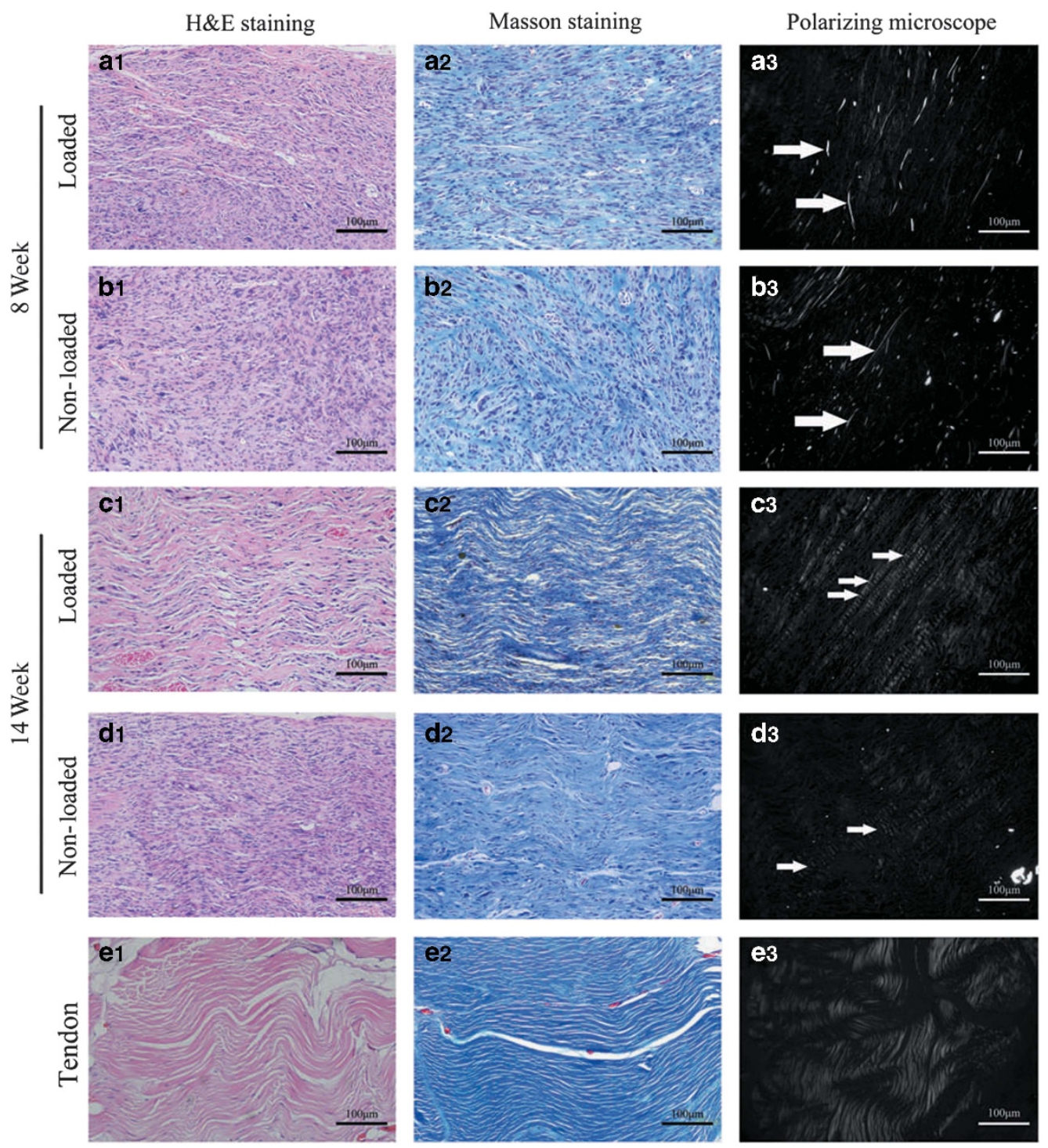

Figure 4 Histological and polarizing microscopic evaluations of tendons engineered with DPSCs. (a-d) H\&E staining, Masson staining, and polarizing microscopic images of the tendons engineered at weeks 8 and 14 postimplantation. (a3, b3) The arrows indicate undegraded PGA fibres. (e) Masson staining and polarizing microscopic images of the native human tendon. Original magnifications: $\times 200 ;$ bar $=100 \mu \mathrm{m}$. DPSCs, dental pulp stem cells; H\&E, haematoxylin and eosin; PGA, polyglycolic acid; W, week. 
8 Week
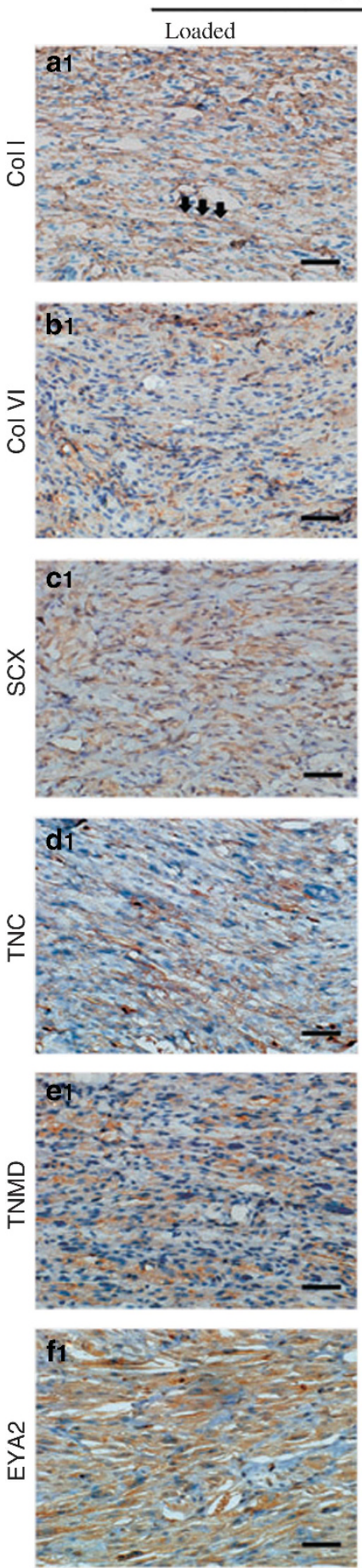
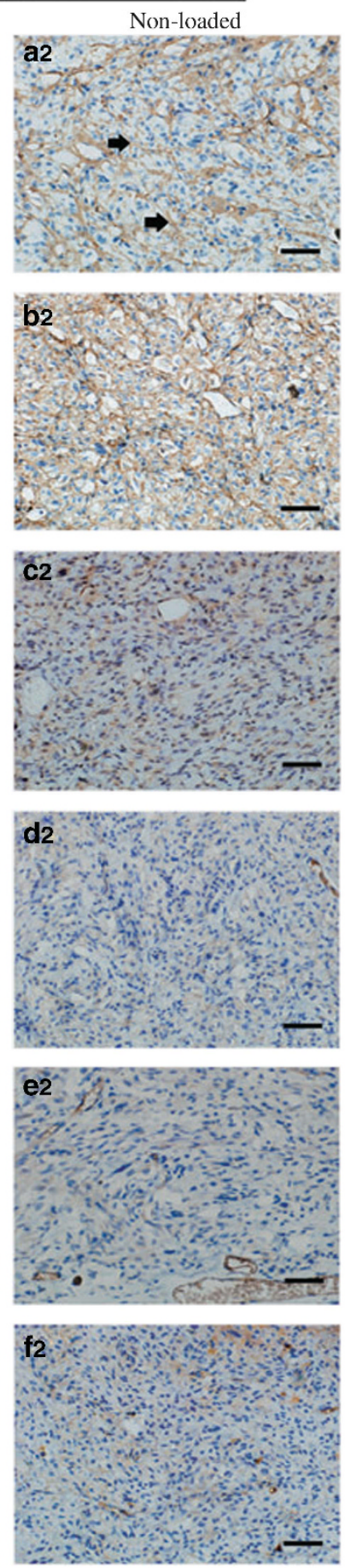

14 Week
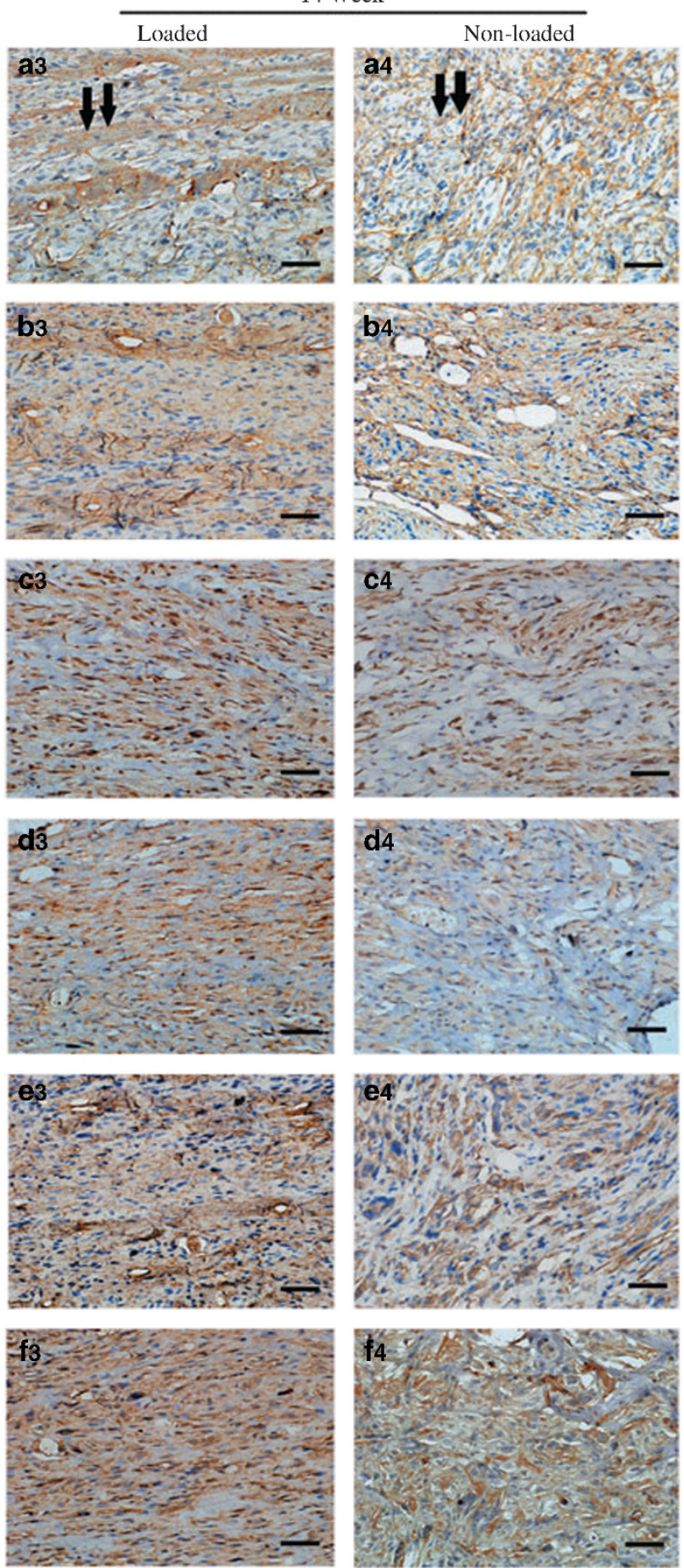

Figure 5 Immunohistochemical staining reveals the expression of crucial tendon-related molecules in the tendons engineered by DPSCs. (a) col I; (b) Col VI; (c) SCX; (d) TNC; (e) TNMD; (f) EYA2. Col I exhibited more organized expression (aligned or bundled) (arrows in al and a3) in the loaded groups than that in the unloaded groups after 8 and 14 weeks postimplantation. The other molecules exhibit an expression pattern similar to the loaded groups. Original magnification: $\times 400$; bar $=50 \mu \mathrm{m}$. DPSCs, dental pulp stem cells; EYA2, eye absent 2; SCX, scleraxis; TNC, tenascin-C; TNMD, tenomodulin.

resulted in a relatively compact tissue and with longitudinally aligned cells relative to those of the group without loading (Figure 4b1). The collagen maturation level was determined by polarized microscopy. As shown in Figure 4a3 and 4b3, immature collagen structure could be detected with weak light reflection in both groups. In addition, some undegraded PGA fibre residues could be observed by optical microscopy (white arrow). After 14 weeks of mechanical loading in vivo, both $\mathrm{H} \& \mathrm{E}$ and Masson staining indicated that a much more mature tissue structure was formed with a composition of mature collagen fibres (in wave patterns) and cellular components 
(exhibiting spindle-shaped nuclei) with proper cell/collagen ratio (Figure 4c2, also shown in Supplementary Data S5). Few mature collagen fibre structures with white colour were observed, and no PGA residues remained (Figure 4c3). By contrast, without effective loading, the tissues that formed exhibited an immature tissue structure characterized by loosely deposited collagen fibres that were randomly arranged and apparently disorganized (Figure 4d1). Moreover, some randomly arranged collagen fibres appeared at the edge of the tissue, and immature collagen structures could also be detected (Figure 4d3).

\section{Expression of tendon-related ECM markers in the regenerative tendon tissues}

To further characterize tendon-like tissue formation by DPSC-PGA constructs under mechanical stimulation, immunohistochemistry staining for collagens I and VI, SCX, TNMD, TNC, and EYA2 were performed in in vivo-formed tissue constructs at weeks 8 and 14 after implantation. These tendon-related proteins were all expressed in the native tendons (Figure 1). As expected, the amount of protein was more pronounced in the loaded group than in the non-loaded groups at both weeks 8 and 14. Similar trends in gene expression of these tendon markers were detected in the DPSCs cultured on PGA scaffolds under mechanical stimulation in vitro. The expression of collagens I and VI was positive at week 8 in both groups (Figure 5a1) and became stronger at week 14 (Figure 5a3). Similarly, the expressions of SCX, TNMD, TNC, and EYA2 were weak in the non-loaded group (Figure 5(c-f)2) but increased significantly at week 14 . Interestingly, the expression of these tendon-related markers in the loaded group was much stronger at week 8 (Figure 5(c-f)1) and 14 (Figure 5(c-f) 3 and Supplementary Data S5) than those in the nonloaded groups at weeks 8 (Figure 5(c-f)2) and 14 (Figure 5(c-f)4), indicating that more mature tendon-like markers in loaded DPSCPGA tissue constructs were expressed.

\section{DISCUSSION}

Tissue engineering has been a promising treatment strategy in tendon repair during the past decades. The cell source is one of the key elements in tissue engineering. Tenocytes are highly differentiated cells; phenotype drift and functional loss of these cells is often observed during in vitro cell expansion, precluding their use as a practical cell source. Much effort has been made to search for a replacement of tenocytes for tendon regeneration and repair. MSCs show promise as an alternative cell source for tendon regeneration. Several animal and clinical studies have confirmed that the application of MSCs can enhance tendon repair and regeneration. ${ }^{12}$ However, harvesting these autologous MSCs may cause injury and impairment at the donor site and potential ectopic ossification. DPSCs are easily available from discarded biological samples (such as extracted teeth) or cryopreserved dental pulp, and their self-renewal and multilineage differentiation potential provide advantages that make them a favourable option over other types of stem cells. ${ }^{13-14}$ In our present study, therefore, we used DPSCs and present them as a novel cell source for tissue engineering of tendon-like tissue by incorporating human DPSCs within 3-D aligned PGA scaffolds under mechanical stimulation.

Dental stem cells in dental-related tissues are derived from neural crest cells and exhibit a high proliferative rate, high viability, and multipotency. The DPSCs used in the present study were obtained from the dental pulp. They are MSCs that display self-renewal and odontoblastic differentiation abilities but exhibit a higher frequency of colony formation than that of bone marrow stem cells. ${ }^{9}$ As shown by our studies and others', DPSCs are multipotent and capable of differentiating into odontoblasts, chondrocytes, melanocytes, pancreatic cells, osteoblasts, and adipocytes; ${ }^{15-16}$ moreover, they express CD146 and Stro1, which are regarded as MSC markers. ${ }^{17-18}$

Dental pulp consists of connective tissue, mainly collagens and some NCPs, which is also supported by our present results. Surprisingly, some tendon-related markers, such as SCX, EYA2, TNC, and TNMD, are also expressed in the dental pulp, especially in the odontoblastic layer. It is well known that odontoblasts, with their high secretory capacity, represent the main differentiation direction for dental pulpderived cells. They produce and secrete collagen I into the extracellular space and form the predentinal interface. In addition to collagenous components, odontoblasts also synthesize NCPs of the ECM in predentin and dentin, such as dentin sialophosphoprotein, dentin matrix acidic phosphoprotein, decorin, and biglycan. Collagens and some NCPs constitute not only the predentinal interface but also the tendon matrix. Therefore, the present finding that dental pulp tissue expresses these tendon-related markers leads us to speculate that dental pulp-derived cells may have the potential to differentiate into mature tenogenic cells (secreting collagens and NCPs) under an appropriate microenvironment.

The cell types in dental pulp include fibroblasts, odontoblasts, pericytes, endothelial cells, and undifferentiated mesenchymal cells, among others. DPSCs, as undifferentiated mesenchymal cells, can differentiate into fibroblasts and odontoblasts and have a crucial role in wound-healing process in the dental pulp. We showed that DPSCs could express collagens I and VI but rarely express tendon-related proteins in vitro, such as SCX, EYA2, and TNC, when cultured in 2-D culture dishes. The mechanical microenvironment can affect cell proliferation, migration, differentiation, and apoptosis, as well as tissue development. ${ }^{19}$ Different types of mechanical stimuli have been widely applied to tissue engineering of tendons. ${ }^{20-21}$ We used custom-fabricated springs and PGA scaffolds modified from the previous work ${ }^{22-23}$ to apply static stretch to DPSCs in vitro. We found that tendon-related gene expression of collagens I and VI, SCX, EYA2, and TNC was upregulated in these engineered constructs during the early culturing period. Our present finding of upregulation of tendonrelated gene expression by static stretch for DPSCs (a type of MSC) is supported by many related research studies. Kuo et al. ${ }^{21}$ found that static strength could upregulate SCX and collagen III consistently in bone marrow stem cells in collagen gel. ${ }^{21}$ Similarly, Scott et al. ${ }^{24}$ demonstrated that the mRNA level of SCX of multipotent $\mathrm{C} 3 \mathrm{H} 10 \mathrm{~T} 1 / 2$ in a $3-\mathrm{D}$ collagen environment increased dramatically over the first week with static load alone. ${ }^{24}$ However, cell differentiation induced by mechanical stretch can vary by the type and the duration of mechanical force in addition to differences between cell types. Some researchers have demonstrated that cyclic tensile strain or pulsating fluid shear stress promoted odontoblastic differentiation of human DPSCs, ${ }^{25-26}$ whereas other reports have indicated that uniaxial stretch increases proliferation while inhibiting osteogenic and odontogenic differentiation of DPSCs. ${ }^{27-28} \mathrm{~A}$ previous study indicated that static strain or even dynamic stretch in vitro may not be an optimal niche for maturation and functional development of engineered tendons; ${ }^{29}$ therefore, it is notable to observe the tendon-like tissue differentiation of DPSCs in vivo. The current animal model in our work is commonly used for regenerating ectopic tendon in vivo. ${ }^{29-30}$ The fastening of a cell-scaffold construct to the mouse back fascia provides a relatively low oxygen tension area and a constant tension platform, thus facilitating tendon formation in vivo. Through 
immunostaining (of collagens I and VI, SCX, TNMD, TNC, and EYA2) and polarized microscopy, we found that DPSCs could be differentiated into tenogenic-like cells and form more matured tendon-like tissues under mechanical loading in the mouse model. Similar to our results, Chen et al. ${ }^{30}$ found that mouse skeletal muscle-derived cells with aligned PGA formed functional engineered tendon tissue. ${ }^{27}$ Another previous study demonstrated the formation of ectopic tendon in a similar mouse model by the engineered tendon of a human embryonic stem cell sheet, especially for those cells genetically modified by SCX. ${ }^{31}$ This evidence indicates that the nude mice back is an ideal microenvironment that can provide constant mechanical stretch and facilitate in vivo tendon formation. Therefore, our current results confirm the hypothesis that DPSCs, as a source of MSCs, may have the potential to differentiate down the tenogenic lineage in a defined microenvironment and form tendon-like tissue in vivo.

\section{CONCLUSIONS}

This study demonstrated that human DPSCs could be a potential cell source for the reconstruction of tendon-like tissues under mechanical stimulation. This tissue engineering of tendon is necessary for further studies on the mechanical properties and maturation of tendon to optimize therapy outcomes in tendon injuries. This preliminary study is also intended to motivate the initiation of subsequent clinical studies.

\section{ACKNOWLEDGEMENTS}

This work was supported by the Natural Science Foundation of China (81171470 and 81100761), the key clinical specialty discipline construction programme of Fujian, China, and the Key Project of Science and Technology Bureau of Jiangsu Province (BL2013002). We thank Dr Sheng-Bo Pan, Dr Ling-Ling Liu, Dr Dan-Hong Zhuang and Dr Hao Yu (Key Laboratory of Stomatology, Fujian Province University), Dr Bo Chen and Dr Guang-Dong Zhou (National Tissue Engineering Research and Development Center) and Dr Yi-De Huang and Dr Xue-Fen Hu (Fujian Key Laboratory of Developmental and Neurobiology) for their assistance in animal work and laboratory experiments.

1 Liu $\mathrm{H}$, Zhu S, Zhang $\mathrm{C}$ et al. Crucial transcription factors in tendon development and differentiation: their potential for tendon regeneration. Cell Tissue Res 2014; 356(2): 287-298.

2 Schweitzer R, Chyung JH, Murtaugh LC et al. Analysis of the tendon cell fate using scleraxis, a specific marker for tendons and ligaments. Development 2001; 128(19): 3855-3866.

3 Moshaverinia A, Xu X, Chen $\mathrm{C}$ et al. Application of stem cells derived from the periodontal ligament or gingival tissue sources for tendon tissue regeneration. Biomaterials 2014; 35(9): 2642-2650.

4 Alberton $\mathrm{P}$, Popov $\mathrm{C}$, Pragert $\mathrm{M}$ et al. Conversion of human bone marrow-derived mesenchymal stem cells into tendon progenitor cells by ectopic expression of scleraxis. Stem Cells Dev 2012; 21(6): 846-858.

5 Aslan H, Kimelman-Bleich N, Pelled G et al. Molecular targets for tendon neoformation. J Clin Invest 2008; 118(2): 439-444.

6 James R, Kumbar SG, Laurencin CT et al. Tendon tissue engineering: adipose-derived stem cell and gdf-5 mediated regeneration using electrospun matrix systems. Biomed Mater 2011; 6(2): 025011.
7 Chong AKS, Ang AD, Goh JC et al. Bone marrow-derived mesenchymal stem cells influence early tendon-healing in a rabbit Achilles tendon model. J Bone Joint Surg Am 2007: 89(1): 74-81.

8 Gronthos S, Mankani M, Brahim J et al. Postnatal human dental pulp stem cells (DPSCs) in vitro and in vivo. Proc Natl Acad Sci USA 2000; 97(25): 13625-13630.

9 Huang GTJ, Gronthos S, Shi S. Mesenchymal stem cells derived from dental tissues vs. those from other sources: their biology and role in regenerative medicine. $J$ Dent Res 2009; 88(9): 792-806.

10 Verma K, Bains R, Bains VK et al. Therapeutic potential of dental pulp stem cells in regenerative medicine: an overview. Dent Res J (Isfahan) 2014; 11(3): 302-308.

11 Linde A. The extracellular matrix of the dental pulp and dentin. J Dent Res 1985; 64: 523-529.

12 Ahmad Z, Wardale J, Brooks R et al. Exploring the application of stem cells in tendon repair and regeneration. Arthroscopy 2012; 28(7): 1018-1029.

13 Altman GH, Horan RL, Martin I et al. Cell differentiation by mechanical stress. FASEB J 2002; 16(2): 270-272.

14 Gronthos S, Brahim J, Li W et al. Stem cell properties of human dental pulp stem cells. $J$ Dent Res 2002; 81(8): 531-535.

15 Stevens A, Zuliani T, Olejnik C et al. Human dental pulp stem cells differentiate into neural crest-derived melanocytes and have label-retaining and sphere-forming abilities. Stem Cells Dev 2008; 17(6): 1175-1184.

16 Ishkitiev N, Yaegaki K, Kozhuharova A et al. Pancreatic differentiation of human dental pulp CD117+ stem cells. Regen Med 2013; 8(5): 597-612.

17 Miura M, Gronthos S, Zhao M et al. SHED: Stem cells from human exfoliated deciduous teeth. Proc Natl Acad Sci USA 2003; 100(10): 5807-5812.

$18 \mathrm{Xie} \mathrm{H}$, Liu H. A nove mixed type stem cell pellet for cementum/periodontal ligament-like complex. J Periodontol 2012; 83(6): 805-815.

19 Ahmed WW, Kural MH, Saif TA. A novel platform for in situ investigation of cells and tissues under mechanical strain. Acta Biomater 2010; 6(8): 2979-2990.

20 Butler DL, Juncosa-Melvin N, Boivin GP et al. Functional tissue engineering for tendon repair: a multidisciplinary strategy using mesenchymal stem cells, bioscaffolds, and mechanical stimulation. J Orthop Res 2008; 26(1): 1-9.

21 Kuo CK, Tuan RS. Mechanoactive tenogenic differentiation of human mesenchymal stem cells. Tissue Eng Part A 2008; 14(10): 1615-1627.

22 Cao D, Liu W, Wei $X$ et al. In vitro tendon engineering with avian tenocytes and polyglycolic acids: a preliminary report. Tissue Eng 2006; 12(5): 1369-1377.

23 Deng D, Liu W, Xu F et al. Engineering human neo-tendon tissue in vitro with human dermal fibroblasts under static mechanical strain. Biomaterials 2009; 30(35): 6724-6730.

24 Scott A, Danielson P, Abraham T et al. Mechanical force modulates scleraxis expression in bioartificial tendons. J Musculoskelet Neuronal Interact 2011; 11(2): 124-132.

25 Lee SK, Lee CY, Kook YA et al. Mechanical stress promotes odontoblastic differentiation via the heme oxygenase-1 pathway in human dental pulp cell line. Life Sci 2010; 86(3/4): 107-114.

26 Kraft DC, Bindslev DA, Melsen B et al. Mechanosensitivity of dental pulp stem cells is related to their osteogenic maturity. Eur J Oral Sci 2010; 118(1): 29-38.

27 Hata M, Naruse K, Ozawa S et al. Mechanical stretch increases the proliferation while inhibiting the osteogenic differentiation in dental pulp stem cells. Tissue Eng Part $A$ 2013; 19(5/6): 625-633.

28 Cai X, Zhang Y, Yang X et al. Uniaxial cyclic tensile stretch inhibits osteogenic and odontogenic differentiation of human dental pulp stem cells. J Tissue Eng Regen Med 2011; 5(5): 347-353.

29 Wang B, Liu W, Zhang Y et al. Engineering of extensor tendon complex by an ex vivo approach. Biomaterials 2008; 29(20): 2954-2961.

30 Chen $\mathrm{B}$, Wang $\mathrm{B}$, Zhang WJ et al. In vivo tendon engineering with skeletal muscle derived cells in a mouse model. Biomaterials 2012; 33(26): 6086-6097.

31 Chen X, Yin Z, Chen JL et al. Force and scleraxis synergistically promote the commitment of human ES cells derived MSCs to tenocytes. Sci Rep 2012; 2: 977.

(c) (i) $\Theta$ This work is licensed under a Creative Commons AttributionBY NC ND NonCommercial-NoDerivs 4.0 International License. The images or other third party material in this article are included in the article's Creative Commons license, unless indicated otherwise in the credit line; if the material is not included under the Creative Commons license, users will need to obtain permission from the license holder to reproduce the material. To view a copy of this license, visit http:// creativecommons.org/licenses/by-nc-nd/4.0/

(C) The Author(s) 2016

Supplementary Information for this article can be found on the International Journal of Oral Science website (http://www.nature.com/ijos) 